\title{
Prevalencia de la violencia escolar EN PREADOLESCENTES DE CÚCUTA ${ }^{1}$
}

\section{Prevalence of School Violence in Preadolescents of Cúcuta}

\author{
Yijadd Ordoñez Yaber ${ }^{2}$, Diany Ariza Takemiche ${ }^{3}$
}

Recibido: 2018-09-14 Aceptado: 2018-12-13

\begin{abstract}
Resumen: Este artículo de tipo investigativo identifica la prevalencia de la violencia escolar respecto a los agentes en el rol de observador, víctima o agresor, con una metodología no experimental transeccional, descriptiva, con una muestra de 196 participantes entre 11 a 13 años de edad en centros educativos públicos, donde se administró el cuestionario de Violencia Escolar. Se encuentra como resultado que la proporción de los agentes de la violencia escolar de la comuna uno de la ciudad de San José de Cúcuta es de un 38\%, determinando una diferencia rol observador, víctima o agresor. A partir de los resultados se puede inferir que los roles de la violencia escolar se presentan de manera simultánea.
\end{abstract}

Palabras clave: observador, víctima, agresor, contexto educativo.

\begin{abstract}
This investigative-type article identifies the prevalence of school violence regarding to the agents, in the role of observer, victim or aggressor using a non-experimental transeccional methodology of descriptive type with a sample of 196 participants from between 11 and 13 years old, from 4 different public educational centers where a school violence questionnaire was administered, finding as a result that the proportion of the school's violence agents in the "commune 1 " of the population of San José de Cúcuta is 38\%, determining a difference according to the role (Observer, victim or aggressor) from results it can be inferred that the roles of school violence are presented simultaneously.
\end{abstract}

Keywords: observer, victim, aggressor, educational context.
Para citar este artículo en APA: Ordoñez, Y., y Ariza, D. (2018). Prevalencia de la violencia escolar en preadolescentes de Cúcuta. Revista de Psicologia Universidad de Antioquia, 10(2), 37-52. DOI: 10.17533/ udea.rp.v10n2a02
Artículo de investigación.

2. Doctoranda en Educación de la Universidad Pedagógica Libertadores. Profesora Universidad de Pamplona, Norte de Santander, Colombia. Correo: yijadd@ hotmail.com; https://orcid.org/0000-0001-7999-7760.

3. Psicóloga, estudiante de especialización en Práctica Pedagógica, Universidad Francisco de Paula Santander. Correo: diamatus1994@gmail.com; https://orcid. org/0000-0001-9116-5561 


\section{Introducción}

El problema de esta investigación se centra en el fenómeno de la violencia escolar — ve-, por lo que resulta ineludible determinar los perfiles en los agentes: agresor, víctima y observador. Los trabajos de Smith, Morita, Junger, Tas, Olweus, Catalano y Slee (1999) muestran un interés por estudiar la incidencia y prevalencia conducentes en el maltrato entre iguales, razón es necesario visibilizar y comprender la violencia esporádica de la persistente, debido a que la influencia del término bullying y su relación con los centros escolares ha favorecido el ocultamiento de otras formas de violencia (Amaury, García y Martínez, 2015).

La violencia escolar es un fenómeno de salud pública y se puede comprender como una serie de comportamientos concurrentes dentro de las aulas de clases caracterizados por la agresión entre iguales y entre estudiantes y profesores. Tales ataques se presentan de manera bidireccional y sus manifestaciones se dan a través de componentes físicos, verbales y psicológicos. Dichos comportamientos impiden el proceso de enseñanza-aprendizaje, pues afectan las relaciones dentro y fuera del aula de clases (Valadez, como se cita en Rincón, 2017).

La relación entre ve y el desarrollo que presentan los estudiantes según la etapa en que se encuentren — niñez o adolescencia y juventud-, genera cambios a nivel psicológico que deben ser regulados y equilibrados por el estudiante $\mathrm{u}$ orientado por otros. Un comportamiento violento presenta emociones alteradas, tendencias a llamar la atención, y puede derivar en una posterior integración a pandillas donde buscan canalizar esta situación, pero de forma no controlada. Esto lleva a que la institución implemente medidas correctivas caracterizadas por la exclusión y la discriminación contra el estudiante, contrario a un proceso de intervención enfocado en la disminución de la violencia (Woolfolk, como se cita en Erazo, 2010).

La palabra violencia suele ser empleada para referirse a conductas agresivas que van más allá de lo «natural» —en un sentido adaptativo—, caracterizadas por su fuerza, intensidad, daño, así como por su aparente carencia de justificación, esto es, su tendencia meramente ofensiva contra el derecho y la integri- 
dad de un ser humano, la cuál puede ser física, psicológica o moral (Carrasco y González, 2006).

En cuanto a la definición de la violencia escolar, ésta abarca diversas perspectivas que dan apertura a unos rasgos y características propias de los individuos que están inmersos en ella, por lo tanto, tal violencia se define según el tipo de comportamiento; en este caso, pueden ser físicos, sexuales o emocionalmente abusivos. Este último ejemplo ocurre cuando un niño, niña, adolescente o grupo, directa o indirectamente, trata mal, daña o mata a otro u otros. De esta forma, existe un punto de consenso que define estas conductas como un acto de agresión física, moral o institucional dirigido contra la integridad, el cual es considerado como violento (Abramovay y Rua, 2002; Castillo y Pacheco, 2008; Hoyos, Aparicio y Córdoba, 2005; Unesco, como se cita en Romera, Alamillo y Ortega, 2011).

Según la Organización Mundial de la Salud (OMS, 2016), las repercusiones no mortales de la violencia en los jóvenes tienen implicaciones graves que afectan las diferentes áreas de las personas — sus componentes físico, psicológico y social—, y constituye una problemática de salud pública debido a que ésta encarece los servicios sanitarios, sociales y jurídicos, y genera improductividad juvenil. Igualmente, por cada joven que muere por causas violentas, otros sufren lesiones que requieren tratamiento hospitalario, lo cual causa afectación familiar.

Asimismo, el resultado que produce la violencia en la niñez y la adolescencia está relacionado con el déficit de atención, trastornos de la conducta, delincuencia, consumo de sustancias psicoactivas, bajo nivel académico y de compromiso con las instituciones educativas, entre otros fenómenos. La oms, las agencias especializadas en educación, infancia-adolescencia o salud y los organismos multilaterales como la Organización de las Naciones Unidas para la Educación y la Cultura - Unesco-y el Fondo de las Naciones Unidas para la Infancia —Unicef_- se han ocupado reiteradamente de llamar la atención sobre un tema de preocupación y presencia creciente como la violencia escolar, y de proponer respuestas a los conflictos que ésta provoca (Monclús, como se cita en Díaz, 2016). 
La presente investigación aborda la problemática de la violencia escolar y se propone identificar su prevalencia según sus agentes —observador, víctima y agresor- en estudiantes preadolescentes de 11 a 13 años, y que cursan grado sexto en colegios públicos de carácter mixto de la comuna uno en la ciudad de Cúcuta. En este sentido, este estudio constituye un aporte a las instituciones educativas respecto a la caracterización e identificación de esta problemática de acuerdo con sexo, rol y edad.

Lo anterior se contrasta con lo planteado por Gázques, Peréz, Carrión y Santuiste (2010), quienes afirman qué al formular programas en las escuelas como método de prevención de la violencia escolar dentro del sistema educativo colombiano, es necesario definir los datos del número de conflictos, esta problemática conlleva a un deterioro del clima escolar y convivencia por lo que es un argumento suficiente en cualquier reflexión acerca de la calidad educativa.

\section{Metodología}

Este estudio de enfoque cuantitativo se enmarcó en una investigación no experimental de carácter transeccional de tipo descriptivo.

\section{Población}

Este proyecto se desarrolló en Cúcuta, la cual cuenta con una población estudiantil de 11180 estudiantes de grado sexto (Secretaria de Educación Municipal, 2017).

Para poder investigar se realizó la solicitud a la Secretaria de Educación Municipal Cúcuta y así obtener datos de las instituciones educativas de carácter público; posteriormente se procedió a sectorizar las instituciones educativas y a partir de los datos recolectados se establecieron los siguientes criterios de delimitación para el desarrollo de la investigación. 
Tabla 1. Criterios de delimitación

\begin{tabular}{|l|l|l|l|l|}
\hline Colegios & Comuna & Jornada & Grados & Edad \\
\hline Públicos mixtos & Uno & Tarde & $6^{\circ}$. & De 11 a 13 años \\
\hline
\end{tabular}

Teniendo en cuenta estos criterios de delimitación se redujo la población a un $5 \%$, lo cual equivale a 532 estudiantes, con un error del $5 \%$ y un nivel de confianza del $95 \%$, distribuidos en las cuatro instituciones educativas. Seguidamente, se hizo un proceso de muestreo estratificado y aleatorio simple ejecutado por el programa stats, dando como resultado una muestra total de 196 estudiantes.

Tabla 2. Muestras de las listas oficiales de las instituciones educativas seleccionadas

\begin{tabular}{|c|c|c|c|c|c|}
\hline \multirow[t]{2}{*}{ Estrato por giro } & \multirow[t]{2}{*}{ Instituciones educativas publicas } & \multirow[t]{2}{*}{ Población } & \multicolumn{3}{|c|}{ Muestra } \\
\hline & & & $\mathrm{H}$ & M & $\mathrm{Nh}$ \\
\hline 1 & Colegio Sagrado Corazón de Jesús la Salle & 276 & 47 & 26 & 73 \\
\hline 2 & Instituto Técnico Nacional de Comercio & 71 & 21 & 20 & 41 \\
\hline 3 & Colegio San José de Cúcuta & 156 & 27 & 33 & 60 \\
\hline 4 & Colegio Antonio Narińo & 29 & 12 & 10 & 22 \\
\hline Total & \multicolumn{2}{|c|}{$\mathbf{N}=532$} & \multicolumn{2}{|c|}{$\mathrm{n}=$} & 196 \\
\hline
\end{tabular}

\section{Instrumento de recolección}

La prueba psicométrica de violencia escolar — ver tabla 3- tiene como objetivo evaluar los agentes de la violencia escolar y fue aplicada en estudiantes entre los 11 y 13 ańos. Tiene una duración de 45 minutos. Consta de 38 reactivos que están distribuidos por tres secciones: agresor - 12 ítems - victima -12 ítems-, observador - 12 ítems- y dos preguntas control. La calificación de los ítems es de 0 a 1 puntos y en la sumatoria si la persona puntúa entre 0 a 5 , el agente no presenta la conducta y entre 6 a 12 si cumple con un rol en la violencia escolar. Cada pregunta está orientada en tres componentes: físico, verbal y psicológico, para determinar el perfil. 
Tabla 3. Descripción de los items de la prueba psicométrica de violencia escolar

\begin{tabular}{|c|c|}
\hline $\mathrm{N}^{\circ}$. Ítems & Pregunta \\
\hline 1 & Acepto las diferencias físicas o mentales que tienen los profesores \\
\hline 2 & Golpeo a mis compañeros \\
\hline 3 & Si un compañero me molesta le discuto con rapidez \\
\hline 4 & Algún compañero me golpea \\
\hline 5 & Lidero peleas dentro o fuera del salón de clases \\
\hline 6 & Me siento maltratado físicamente por el profesor \\
\hline 7 & Observo que mis compañeros maltratan físicamente a los profesores \\
\hline 8 & Me burlo del aspecto físico de mis compañeros \\
\hline 9 & Me siento rechazado por algunos compañeros \\
\hline 10 & Noto que mis compańeros se mencionan los defectos físicos \\
\hline 11 & Noto que el profesor amenaza a los estudiantes \\
\hline 12 & Mis compañeros me gritan dentro o fuera del salón de clases \\
\hline 13 & Mis compañeros me ponen sobrenombres \\
\hline 14 & Digo groserías a los profesores cuando les hablo \\
\hline 15 & Pongo sobrenombres a mis compañeros \\
\hline 16 & Los profesores me gritan dentro o fuera del salón de clases \\
\hline 17 & Rechazo a mis profesores por tener diferencias físicas o mentales \\
\hline 18 & Algunos profesores me ponen sobrenombres \\
\hline 19 & Mis compańeros se ponen sobrenombres \\
\hline 20 & Algunos profesores les ponen sobrenombres a mis compañeros \\
\hline 21 & Noto que mis compañeros se hacen gestos humillantes \\
\hline 22 & $\begin{array}{l}\text { Observo que mis compañeros gritan groserías dentro o fuera del salón de clases a los pro- } \\
\text { fesores }\end{array}$ \\
\hline 23 & Hablo con insultos a los profesores \\
\hline 24 & Me doy cuenta que entre mis compañeros se golpean \\
\hline 25 & Me siento rechazado dentro o fuera del salón de clases \\
\hline 26 & Varios de mis compañeros se golpean dentro o fuera del salón de clases \\
\hline 27 & Los profesores maltratan físicamente a algunos compañeros \\
\hline 28 & Mis compañeros me golpean con objetos \\
\hline 29 & Arrojo objetos al profesor \\
\hline 30 & Algunos profesores me golpean \\
\hline
\end{tabular}




\begin{tabular}{|l|l|}
\hline 31 & Insulto a los profesores \\
32 & Amenazo a mis compañeros \\
34 & Observo que algunos de mis compañeros se amenazan \\
35 & Noto que mis compañeros amenazan a los profesores \\
36 & Realizo comentarios humillantes sobre los profesores \\
37 & Los compañeros hacen comentarios desagradables sobre mí \\
38 & Participo en peleas dentro o fuera del salón de clases \\
\hline
\end{tabular}

\section{Procedimiento}

Se realiza el contacto con la Secretaria Municipal, donde se obtuvo el listado de colegios púbicos de la ciudad de Cúcuta para la delimitación y sectorización de la comuna, llegando a ser esta más homogénea a partir de los criterios establecidos. Posteriormente se eleva una autorización a cada institución con base en una solicitud formal documentada para el desarrollo de la investigación.

Luego de que las instituciones autorizaran la aplicación del instrumento para la recolección de datos se procedió a la cumplimentación del cuestionario de manera anónima y voluntaria, el cual se realizó a partir del diligenciamiento del consentimiento informado.

\section{Fase I}

En esta fase se construye la prueba psicométrica o cuestionario Violencia Escolar — ve- Seguidamente se desarrolla un proceso de validación por jueces expertos —nacionales: Mg. en Investigación, PhD en Salud, Mg. en Educación; y un juez internacional experto en trabajo social y psicología clínicacon la finalidad de corroborar la validez del criterio, constructo y contenido de los ítems planteados; ulteriormente, se realiza la prueba de concordancia entre los jueces, obteniendo una puntuación de 0.79 .

Se aplica una prueba piloto con 49 estudiantes de 11 a 13 años de grado sexto de la comuna 8 , los cuales pertenecen a cuatro instituciones educati- 
vas públicas: Instituto Técnico Padre Manuel Briceño Jáuregui Fe y Alegría, Colegio Jaime Garzón, Colegio Pablo Neruda e Institución Educativa Juan Atalaya. Se estimó un tiempo de 60 minutos para su aplicación.

\section{Fase II}

Se lleva a cabo la selección de la muestra y, en consecuencia, la aplicación del cuestionario ve a 196 preadolescentes pertenecientes a cuatro instituciones educativas públicas de carácter mixto, jornada tarde, de la comuna uno de la ciudad de Cúcuta.

\section{Fase III}

Se sistematizan los resultados obtenidos de los cuestionarios y posteriormente se desarrolla el análisis y discusión de los resultados. La información recolectada se analizó a partir de la definición conceptual y operacional de las variables identificadas, las dimensiones, los indicadores e ítems. Se procedió luego a la codificación de los datos, lo cual consistió en darle un valor a cada variable desde la matriz en columnas o filas que representaban las unidades de los participantes. Se seleccionó el programa estadístico spss — media—, se ingresaron los datos, se analizaron por la variable de estudio y por último se prepararon los resultados para su presentación.

\section{Resultados}

Los resultados de la investigación se organizaron de la siguiente manera: en la figura 1 se plantea la distribución general de los agentes de la violencia escolar, en las figuras 2, 3 y 4 se describen los agentes en función del sexo y su rol, la figura 5 en función a la edad y la Figura 6 trata de la distribución de agentes cuando se presenta más de un rol. En la segunda parte se organiza los factores que resultan por cada categoría. Finalmente, se presentan las relaciones que se 
hallaron: la mayor parte del análisis de la información es descriptiva, principalmente de frecuencias, promedios y porcentajes.

Figura 1. Agentes de la violencia escolar

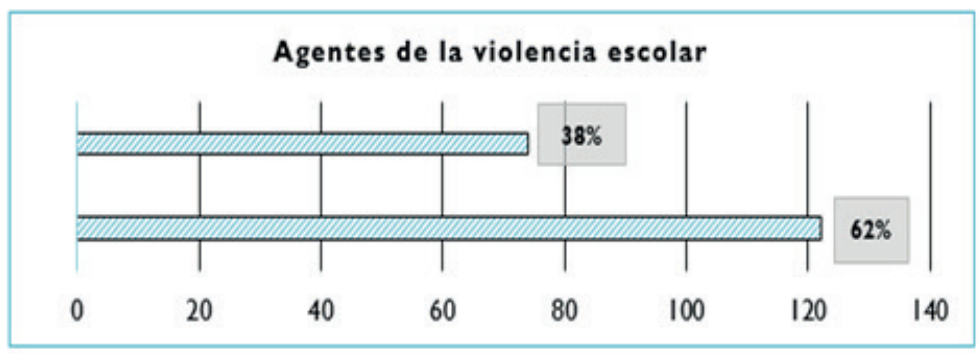

El $38 \%$ corresponde a la categorización de acuerdo con el perfil de los agentes de la violencia escolar de los colegios ubicados en la comuna uno de Cúcuta. Esto equivale a 74 estudiantes que fueron identificados con comportamientos de violencia escolar distribuidos en tres categorías.

Figura 2. Distribución de los agentes de la violencia escolar según el rol

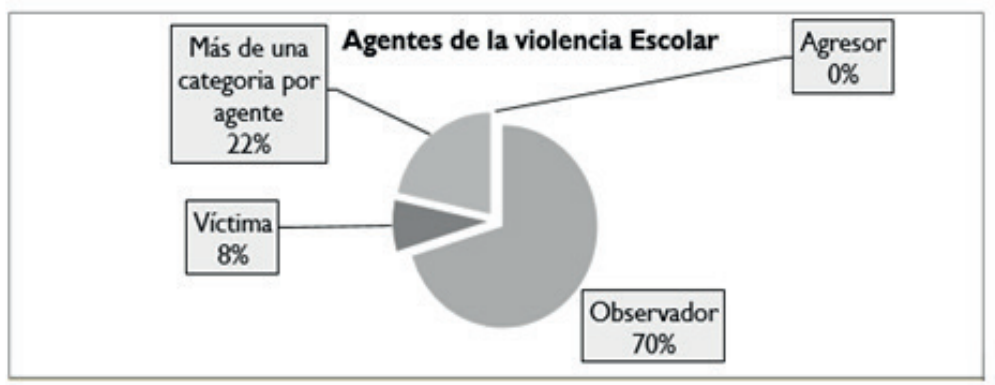

En cuanto a la distribución de los agentes, se identificó que 74 estudiantes presentan comportamientos de violencia escolar según el rol, lo que evidenció la proporción de la prevalencia de los perfiles de los agentes. El 70 \% equivale a 52 estudiantes que son observadores de la violencia escolar, mientras que el $22 \%$ pertenecen a más de una categoría por agente, dado que presentan simultáneamente comportamientos de más de un perfil. El $8 \%$ de los preado- 
lescentes son víctimas, el $0 \%$ son agresores —en cuanto al comportamiento individual一.

Figura 3. Agentes de la violencia escolar en función del sexo

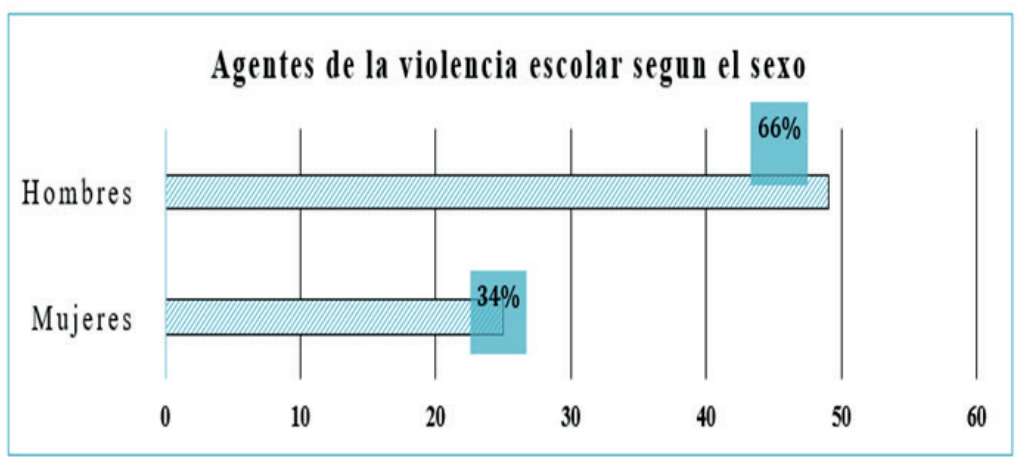

El $34 \%$ de los participantes corresponde a las preadolescentes que son agentes de la violencia escolar, lo cual equivale a 25 estudiantes; en tanto el 66 $\%$ restante a los preadolescentes, lo cual equivale a 49 estudiantes.

Figura 4. Agentes de la violencia escolar en función del sexo y su rol

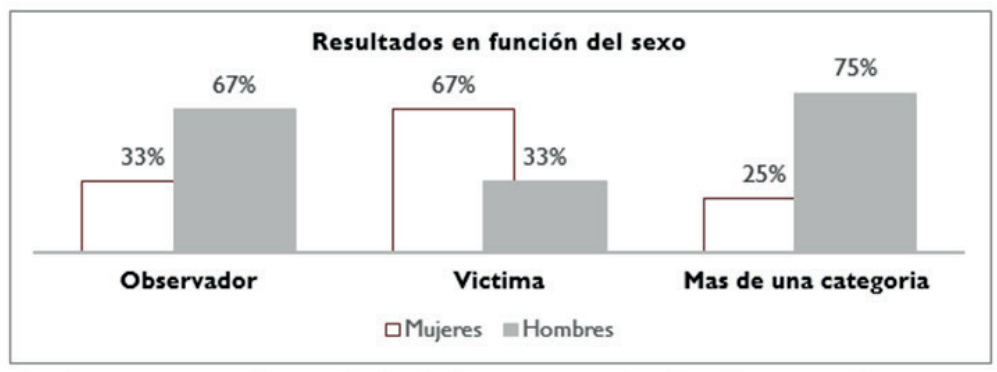

Esta figura representa los resultados de los agentes en función del sexo y rol, con una muestra de 89 mujeres y 107 hombres. El perfil observador, en proporción de prevalencia, equivale a 35 hombres y 17 mujeres, mientras el perfil víctima corresponde a 4 mujeres y 2 hombres. El componente de más de una categoría por perfil corresponde a 12 hombres y 4 mujeres. 
Figura 5. Distribución de los agentes de la violencia escolar según la edad

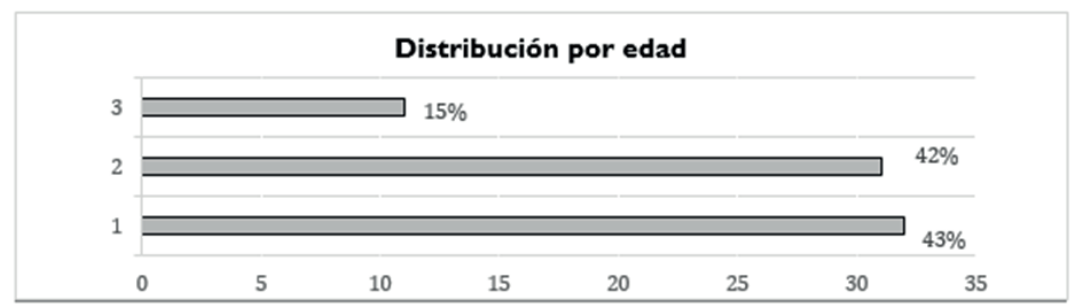

Según esta distribución, entre las edades de participación se analiza que el $43 \%$ de los estudiantes que son agentes de violencia escolar tienen 11 años, el $42 \% 12$ ańos y el $15 \%$ restante 13 ańos.

Figura 6. Distribución de los agentes de la violencia escolar pertenecientes a más de una categoría por perfil

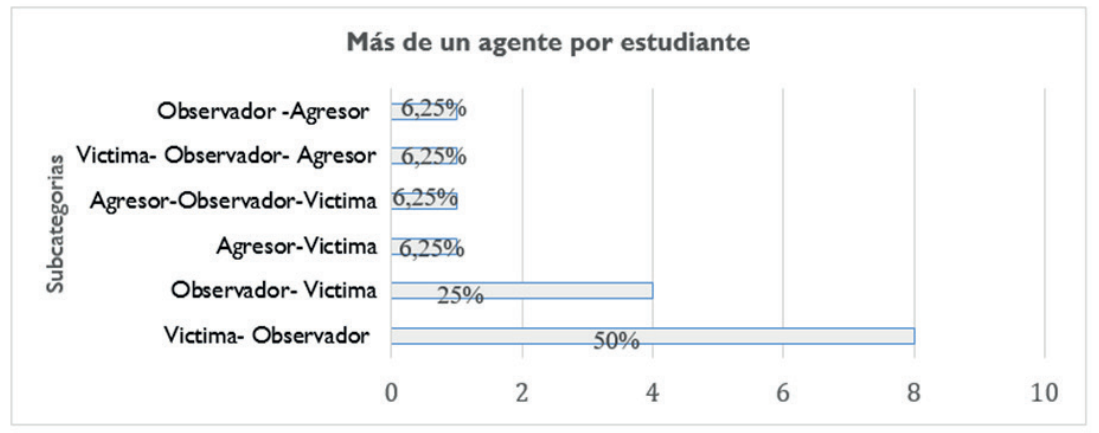

Esta figura representa a los estudiantes que tuvieron puntuaciones suficientes para ubicarse en más de un perfil: el $50 \%$ son preadolescentes con comportamientos prevalentes en la víctima y observador por igual, en tanto el $25 \%$ de los estudiantes tienen comportamientos de observador y víctima; el $6.25 \%$ pertenece a los estudiantes que obtuvieron puntuaciones tanto en agresor como en víctima; el $6.25 \%$ es agresor y observador, el $6.25 \%$ víctima y observador y el $6.5 \%$ es observador-agresor. 


\section{Discusión y conclusiones}

A nivel descriptivo, los resultados de esta investigación muestran la proporción de la prevalencia de la violencia escolar en cuatro instituciones de la comuna uno de la ciudad de Cúcuta. Con base en los datos obtenidos de dichos preadolescentes se pueden corroborar las siguientes hipótesis.

Respecto a la proporción de la prevalencia de los agentes de la violencia escolar, ésta es diferente de acuerdo con el rol —observador, víctima o agresor- Los agentes de la violencia escolar de la comuna uno de Cúcuta corresponde a un $38 \%$. Con una diferencia según el rol, los agentes de violencia escolar se producen con mayor frecuencia en el rol de observador en un $70 \%$ y en las víctimas en un $8 \%$; el $22 \%$ pertenecen a más de una categoría por perfil.

Se corrobora la segunda hipótesis: en los agentes de la violencia escolar se presentan diferencias en función del sexo, pues el $66 \%$ de los hombres ejerce algún rol, mientras que las mujeres ello ocurre en un $34 \%$. La edad con mayor frecuencia de los agentes de la violencia es de 11 años, equivalente a un $43 \%$ de la muestra.

Los resultados en la investigación concuerdan con la teoría y ubican en primer lugar entre los agentes de la violencia escolar a los estudiantes con 11 años en un $43 \%$, seguidos de los estudiantes 12 en un $42 \%$ y los estudiantes de 13 en un $15 \%$. En cuanto a la función del sexo existe variación entre hombres y mujeres. Conforme avanzaban en edad disminuía la agresión física y aumentaban las formas más elaboradas de violencia, tales como la exclusión social y la ridiculización grupal (Orduz, Nieto, Gomezáquira y Luna, 2018).

Se identificaron aquellos estudiantes que experimentan la violencia escolar desde dos o más tipos de agentes simultáneos — víctima-observador, observador-víctima, agresor-víctima- observador-, es decir que no sólo se presentan con un único agente. De los resultados obtenidos en esta investigación se puede deducir que en la muestra estudiada la exposición a la violencia escolar en algún momento parece ser bastante común, y puede ser clasificada en dos o más categorías por estudiante (Wolke, Woods y Stanford, 2001; Salmivalli, 2010). 


\section{Recomendaciones}

Una vez terminado el proyecto de investigación es necesario seguir profundizando en estudios sobre la violencia escolar, ya que es un fenómeno multicausal; se ha de estudiar, pues, la relación de sus componentes y el posible vínculo entre los roles de los agentes.

Es menester seguir avanzando en la realización de estudios que permitan describir los agentes de la violencia en grados superiores como noveno y once para la comprensión evolutiva de los comportamientos y el manejo de las instituciones educativas de carácter público y privado. Esta recomendación se da como propuesta a las instituciones para trabajar este fenómeno por grados y por edad, como una invitación a la prevención de la violencia juvenil y social para fortalecer un modelo de país más pacífico y educado.

A nivel cuantitativo se sugiere seguir identificando la prevalencia de los agentes de la violencia escolar en la ciudad de San José de Cúcuta para tener una base estadística de las comunas y así determinar los índices que predominan en la violencia esporádica o sistemática que ocurre en los salones de clases. Desde los resultados encontrados en la presente investigación es necesaria la creación de una guía educativa sobre la prevención en educadores para que logren identificar los factores relacionados con la violencia escolar.

También se recomienda para el abordaje de esta problemática y su comprensión llevar a cabo estudios de tipo correlacional donde se analicen los estilos parentales de crianza frente a los agentes de la violencia escolar. En la medida en que se puedan comprender las relaciones dadas entre las variables mencionadas, se podrá diseñar programas de intervención integral con el estudiante, los padres y la institución educativa en pro de la convivencia escolar y el fortalecimiento de futuras líneas de investigación.

Se aconseja efectuar intervenciones sobre la estructura de sistemas como la familia, las instituciones y las relaciones sociales para involucrar directamente a los centros educativos una problemática latente que actualmente sufren los preadolescentes e interfiere en el proceso de aprendizaje. Investigadores como Magendzo, Toledo y Rosenfield proponen actividades destinadas a profesores y padres de familia, pues ellos son actores fundamentales para la educación en la convivencia y la paz (como se cita en Rincón, 2017). 


\section{REFERENCIAS}

Alcántara, M. (2009). El bullying: acoso escolar. Innovación y Experiencias Educativas, 16.

Amaury, C., García, M., y Martínez, A. (2015). Bullying y violencia escolar: diferencias, similitudes, actores, consecuencias y origen. Intercontinental Psicología y Educación, 9-38.

Carrasco, O., y González, C. (2006). Aspectos conceptuales de la agresión: definición modelos explicativos. Acción Psicológica, 4(2), 7-38.

Cid, P., Díaz, A., Peréz, V., Torruella, M., y Milady, V. (2008). Agresión y violencia en la escuela como factor de riesgo del aprendizaje escolar. Ciencia y Enfermería, $X I V(2), 21-30$.

Contreras, A. (2013). El fenómeno de bullying en Colombia. Logos Ciencia \& Tecnologia, 4(2), 100-114.

Del Rey, R., y Ortega, R. (2007). Violencia escolar: claves para comprenderla y afrontarla. Escuela Abierta, 10, 77-89.

Delgado, G. (2012). Violencia en la escuela: actores involucrados. Revista de Investigación, 75(36), 53-66.

Díaz, R. (2016). La violencia en la escuela: un análisis desde las revistas indexadas. Seres, Saberes y Contextos, 1, 17-21.

Erazo, O. (2010). Reflexiones sobre la violencia escolar. Revista Psicología GEPU, 1(3), 74-86.

Espelage, D., y Swearer, S. (eds.). (2010). A social-ecological Model for Bullying Prevention and Intervention: Understanding the Impact of Adults in the Social Ecology of Youngsters. En S. R. Jimerson, S. M. Swearer, y D. L. Espelage (eds.), Handbook of Bullying in Schools: An International Perspective (pp. 61-72). New York, ny, us: Routledge/Taylor y Francis Group.

Flórez, G., Villalobos, J., y Londońo, D. (2017). El acompańamiento familiar en el proceso de formación escolar para la realidad colombiana: de la responsabilidad a la necesidad. Revista Virtual de Ciencias Sociales y Humanas, 11(18), 196-220.

Gamboa, S., Alejandro, O., y Muñoz, G. (2017). Violencia en contextos escolares: percepción de docentes sobre manifestaciones de violencia en instituciones en Cúcuta-Norte de Santander. Psicogente, 20(37), 89-98. 
Garaigordobil, M., y Oñederra, J. (2009). Un análisis del acoso escolar desde una perspectiva de género y grupo. Ansiedad y Estrés, 15(2-3), 193-205.

Gázquez, J. J., del Carmen Pérez-Fuentes, M., Carrión, J. J., \& Santiuste, V. (2010). Estudio y análisis de conductas violentas en Educación Secundaria en España. Universitas Psychologica, 9(2), 371-380.

González, R., Mariaca, P., y Arias, T. (2014). Estudio exploratorio del bullying en Medellín. Pensando en Psicología, 10(17), 17-25.

Montagut, A., y Montagut, G. (2014). Representaciones sociales de lo legal en el ámbito escolar a propósito del manual de convivencia escolar. Justicia Juris, 10(1), 66-73.

Navarrete, A., y Ossa, C. (2011). Estilos de crianza y calidad de vida en padres de preadolescentes que presentan conductas diruptivas en el aula (Tesis de maestría). Universidad del Bío-Bio, Chile.

Orduz, F., Nieto, B., Gomezaquira, D. \& Luna, M. (2018). Caracterización del bullying en adolescentes en colegios de Cúcuta. En Andrea Aguilar, Yurley Hernández, Yudith Contreras y Marcela Flórez (Eds). La Investigación Educativa: Reconociendo la escuela para transformar la educación (pp. 162-182). Barranquilla: Ediciones Universidad Simón Bolívar.

Organización Mundial de la Salud [OMS] (30 de septiembre de 2016). Recuperado de http://www.who.int/mediacentre/factsheets/fs356/es/, el 6 octubre de 2017.

Palomero, J., y Fernández, M. (2001). La violencia escolar: un punto de vista global. Revista Interuniversitaria de Formación del Profesorado, 41, 19-38.

Perea, M., Calvo, A., y Anguiano, M. (2010). La familia y la escuela coexistiendo con la violencia escolar. Margen, 58, 1-15.

Rincón, M. (2017). Percepciones de estudiantes y docentes sobre las acciones institucionales de prevención de la violencia escolar en colegios públicos de Cúcuta. Perspectivas, 2(2), 56-72.

Romera, E., Del Rey, R., y Ortega, R. (2011). Prevalencia y aspectos diferenciales relativos al género del fenómeno bullying en países pobres. Psicothema, 23(4), 624-629.

Rodicio, M., e Iglesias, M. (2011). El acoso escolar: diagnóstico y prevención. Madrid: Biblioteca Nueva.

Rodríguez, M. (2010). Factores personales y familiares asociados a los problemas de comportamiento en niños. Escudos de Colombia, 27(4), 437-444. 
Saarento, S. (2015). Conceptualizing and Counteracting the Bullying Dynamic: Classroom and Schoolcontexts Matter (Tesis de grado). Universidad de Turku, Finlandia.

Salmivalli, C. (1999). Participant Role Approach to School Bullying: Implications for Interventions. Journal of Adolescence, (22), 453-459.

Salmivalli, C. (2010). Bullying and the Peer Group: A review. Aggression and Violent Behavior, (15), 112-120.

Sampieri, R., Collado, C., y Baptista, P. (2014). Metodología de la investigación. México: McGraw-Hill / Interamericana Editores.

Secretaría de Educación Municipal (2012). Secretaría de Educación Municipal. San José de Cúcuta: Alcadia Municipal de San José de Cúcuta.

Secretaria de Educación Municipal (2017). Directorio de centros educativos de carácter público. San José de Cúcuta: Secretaría de Planeación y de Desarrollo Educativo.

Smith, P., Morita, Y., Junger-Tas, J., Olweus, D., Catalano, R., y Slee, P. (eds.) (1999). The Nature of School Bullying: A cross-national Perspective. Londres: Routledge.

Torío, S., Peña, J., e Inda, M. (2008). Estilos de educación familiar. Psicothema, 20(1), 62-70.

Torres, E., y Velásquez, A. (2008). Violencia en los colegios de Bogotá: contraste internacional y algunas recomendaciones. Revista Colombiana de Educación, 55, 14-37.

Valadez, F. (2008). Violencia escolar: maltrato entre iguales en escuelas secundarias de la zona metropolitana de Guadalajara. Guadalajara: Colección Salud Materno Infantil.

Veenstra, R., Lindenberg, S., Zijlstra, B., De Winder, A., Verhulst, F., y Ormel, J. (2007). The Dyadic Nature of Bullying and Victimization: Testing a Dual-Perspective Theory. Child Development, 78(6), 1843-1854.

Vela, A. (2015). Del concepto jurídico de familia en el marco de la jurisprudencia constitucional colombiana: un estudio comparado en América Latina. Recuperado de https://repository.ucatolica.edu.co/bitstream/10983/2746/1/DEL\%20 CONCEPTO\%20JURIDICO\%20DE\%20FAMILIA.pdf

Wolke, D., Woods, S., Stanford, K, y Schulz, H. (2001). Bullying and Victimization of Primary School Children in England and Germany Prevalence and School Factors. The British Psychological Society, 92(4), 673-696. 